\title{
El acceso de los hijos de inmigrantes a la Educación Superior en España juna cuestión de origen étnico o de origen social?
}

The Access of the Children of Immigrants to Higher Education in Spain: A Question of Ethnic Origin or Social Origin?

\author{
Damián Herrera Cuesta ${ }^{1}$
}

\section{Resumen}

El presente trabajo se enmarca dentro de los estudios sobre la Dimensión Social de la Educación Superior (DOES). En él se estudia el peso que la posición socioeconómica tiene en el acceso a la Educación Superior de las segundas generaciones en España. Para ello analizamos la probabilidad de que los estudiantes con antecedentes migratorios y diferentes orígenes socioeconómicos realizaran estudios terciarios durante la segunda década del siglo xxi en contraste con la población autóctona de nuevo ingreso. En nuestros resultados encontramos diferencias significativas en el acceso a la universidad entre los jóvenes con edades comprendidas entre los 18 y 21 años, pertenecientes a las segundas generaciones nacidas de matrimonios coétnicos de origen suramericano y norteafricano, lo que podría explicar la actual ralentización de la expansión de la educación terciaria en España.

\section{Palabras clave}

Hijos de inmigrantes, segunda generación, educación superior, origen social, acceso a la universidad.

\section{Abstract}

This work is part of the studies on the Social Dimension of Higher Education (DOES). It studies the weight that socioeconomic position has on access to Higher Education for second generations in Spain. To do this, we analyze the probability that students with migratory backgrounds and different socioeconomic origins carried out tertiary studies during the second decade of the $21^{\text {st }}$ century in contrast to the newly admitted native population. In our results, we found significant differences in access to university among young people between the ages of 18 and 21, belonging to the second generations born of co-ethnic marriages of South American and North African origin, which could explain the current slowdown in expansion of tertiary education in Spain.

\section{Keywords}

1.5 generation, second generation, higher education, social origin, access to university.

\section{Cómo citar/Citation}

Herrera Cuesta, Damián (2021). El acceso de los hijos de inmigrantes a la Educación Superior en España ¿una cuestión de origen étnico o de origen social? Revista de Sociología de la Educación-RASE, 14 (3), 391-406. http://dx.doi.org/10.7203/RASE.14.3.21217. 


\section{Introducción}

En apenas unas décadas, España ha pasado de ser un país emisor de migración internacional a convertirse en un país principalmente receptor. Según los últimos datos facilitados por la Organización Internacional de las Migraciones (OIM, 2020), España forma parte de los veinte primeros países de destino para la población inmigrante. Es sabido que esta situación responde a un momento de intensificación de los movimientos transnacionales de personas producido en las últimas décadas en el marco de la globalización, con la particularidad de que la llegada de inmigrantes a España se produce en un contexto demográfico caracterizado por el envejecimiento de su población y un progresivo declive del volumen de los grupos de personas en edad de trabajar.

La llegada de trabajadores internacionales a España durante los años 90 hizo posible que el saldo vegetativo adoptara un signo favorable contribuyendo al incremento del número de nacimientos, de modo que, en la actualidad, la población española cuenta con varias cohortes nacidas o/y socializadas tempranamente en nuestro país — la denominadas «segunda generación» y «generación 1.5» respectivamente—.

Estas nuevas cohortes nacidas de población inmigrante llegada al país en la década de los 90 representan una parte cada vez más importante de la población española en los últimos años como vemos en el gráfico 1.

Al tiempo que la población autóctona con edades comprendidas entre los 18 y los 21 años presenta una tendencia descendente, la población del mismo grupo de edad con antecedentes migratorios muestra una tendencia ascendente inversamente proporcional (Ver gráfico 1). Sin duda, la eliminación de las barreras que pueda encontrar este conjunto de población española en su proceso de integración social debería ser una importante preocupación para el conjunto de la sociedad.

\section{Gráfico 1. Evolución de la población residente en España con edades comprendidas entre 18 y 21} años según nacionalidad española o extranjera entre 2015 y 2019

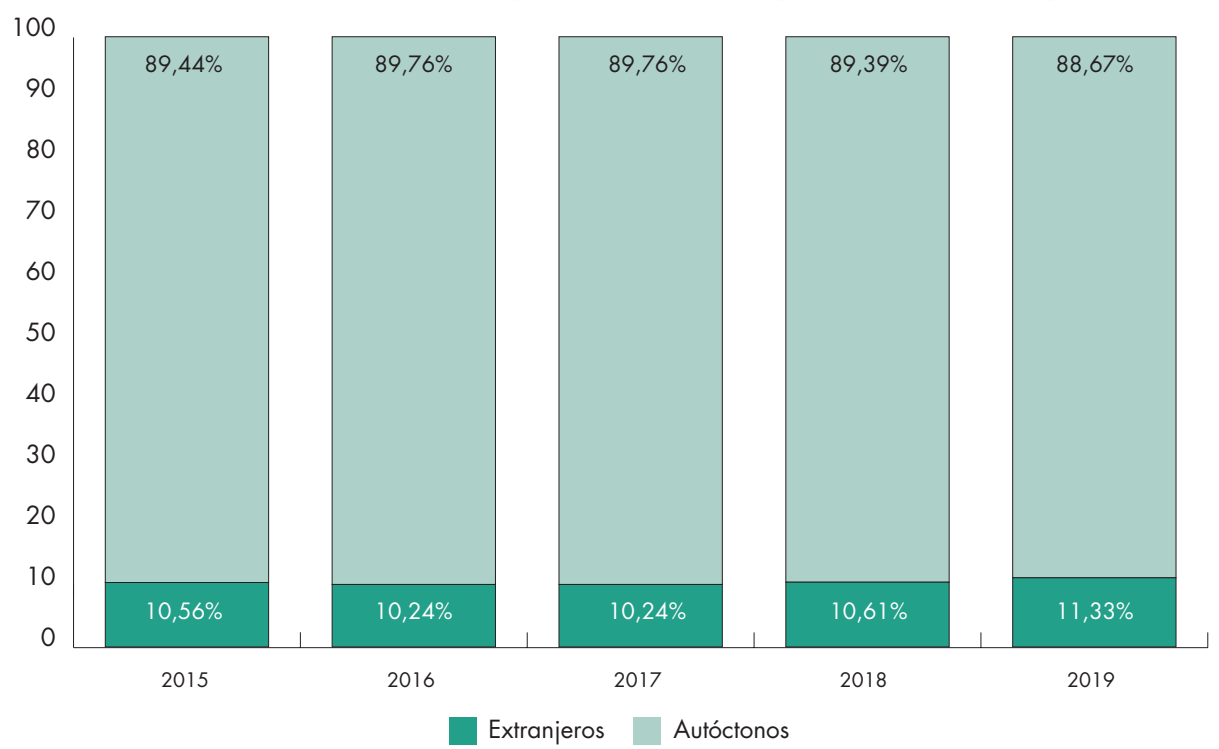

Gráfico de elaboración propia. Fuente: Instituto Nacional de Estadística (INE). 
En general, existe consenso social en torno a la importancia que han tenido en el pasado la expansión de los estudios superiores en las sociedades modernas. También existe un gran consenso científico a la hora de reconocer la existencia de barreras socioeconómicas, muy especialmente desde la crisis económica de 2011 (Herrera, 2017), que impiden o constriñen las oportunidades de acceso a la universidad para una parte de la población. En este sentido, el análisis de la Dimensión Social de la Educación Superior (DOES) en España, debido a la incorporación al sistema educativo de las nuevas cohortes formadas por los hijos de la población inmigrante, debe sumar a aquellos condicionantes que pudieran tener alguna relación con la etnicidad, las barreras socioeconómicas, a fin de poder determinar en qué medida, y de qué modo, ambos aspectos se encuentran conectados.

Teniendo como objetivo general estudiar la relación de los antecedentes migratorios, el origen social de los jóvenes y el acceso a la universidad nuestras preguntas de investigación en el presente trabajo se formulan del siguiente modo:

1. ¿El contexto socioeconómico condiciona la probabilidad de que la población joven con antecedentes migratorios residente en España ingrese en la universidad?

2. Y, de ser así, ¿Comparativamente, el origen social condiciona de igual modo el acceso a la educación superior entre la población joven con antecedentes migratorios que a la juventud autóctona?

Es importante señalar que, entre la población con antecedentes migratorios, incluimos a aquellos hijos de inmigrantes nacidos en España que a través de procesos de naturalización poseen nacionalidad española ( $2^{a}$ generación de matrimonios coétnicos en este estudio), así como también a aquellos individuos que, habiendo nacido en el extranjero, llegaron a España a una edad muy temprana y fueron socializados en el país (Generación 1.5).

Para dar respuesta a las preguntas planteadas se ha llevado a cabo un análisis de regresión logística binaria, técnica que nos permite conocer las influencias y las interacciones simultáneas de las distintas variables introducidas en el análisis. Como variable dependiente dicotómica (sí/no) se tomó la categoría «cursando estudios terciarios», variable que indica quiénes cursan estudios universitarios de primer grado en el momento de la recogida de los datos, es decir, el acceso, no el logro educativo. En un primer paso, se introdujeron como variables objetivas el sexo, la edad de la madre, los estudios y ocupación de los progenitores, el país de nacimiento, los antecedentes migratorios y el tamaño del hogar. Posteriormente se procedió, en un segundo paso, a relacionar la probabilidad del efecto con categorías calculadas a partir del estatus socioeconómico familiar, los antecedentes migratorios y el origen étnico de estos antecedentes.

La presentación de este trabajo se articula a través de los siguientes apartados. En primer lugar, se hace una revisión de la literatura existente en torno a los procesos de integración y el acceso a la universidad de los hijos de inmigrantes, presentando los principales conceptos y enfoques teóricos existentes en la actualidad sobre la materia. Posteriormente, en el apartado metodológico se describe el procedimiento estadístico utilizado. A continuación, se ofrece una síntesis con los principales resultados, dando respuesta a las preguntas formuladas al inicio de la investigación. Finalmente, en un último apartado, se exponen las principales conclusiones obtenidas. 


\section{Marco teórico}

En la literatura sobre la integración de las segundas generaciones y subsiguientes se distinguen dos corrientes de investigación fundamentales. Por un lado, una corriente culturalista centrada en el análisis de los factores socioculturales como principales variables explicativas del proceso de integración /asimilación de la población inmigrante (Navas, Rojas, Pumares, Lozano y Cuadrado, 2010; Álvarez-Sotomayor y Martínez-Cousinou, 2020). Y por otro, una corriente estructuralista que destaca el papel de los factores estructurantes de las relaciones sociales, como los recursos socioeconómicos disponibles por los individuos o el contexto social de oportunidades existente en la sociedad de destino (Rahona y Morales, 2013; Aparicio y Portes, 2014).

Desde el punto de vista culturalista, el éxito de integración depende en último término del grado de asimilación de los patrones culturales dominantes en la sociedad de acogida, entendiendo dicha transición como un proceso dilatado en el tiempo, intergeneracional, y sujeto a pautas definidas de asimilación (idioma, valores y normas sociales, etc.). Dentro de esta perspectiva, en su línea más genuina, se cuentan los trabajos de Gordon (1964) en Estados Unidos. Más recientemente, un enfoque revisado de este punto de vista, lo encontramos en autores como Alba (2005), quién introduce en el estudio de los inmigrantes de segunda generación en Francia, Alemania y los Estados Unidos, la variable psicosocial. Según este autor las mayorías étnicas imponen una distancia social a los inmigrantes y segundas generaciones, resultando de ello una frontera sociológicamente compleja de traspasar para éstos. Estas distancias sociales son específicas en cada sociedad receptora (Alba, 2015).

Los estudios estructuralistas por su parte cubren una amplia variedad de aspectos centrales que tienen como denominador común aquellos factores institucionales y socioeconómicos que condicionan o determinan los resultados del proceso de integración de las segundas generaciones de la población inmigrante (una clasificación extensa puede verse en Aparicio y Portes, 2014, p. 23).

Un enfoque revisado de la perspectiva estructuralista fue planteado en la primera década del siglo xxi por Portes y de Wind (2004). Estos autores proponen la «teoría de la asimilación segmentada» como una formulación integradora para abordar el estudio de la integración social de las segundas generaciones. Adoptando una perspectiva comprensiva, además de los factores de naturaleza estructural recogen también aspectos culturales, como la existencia de una buena disposición del entorno primario hacia los valores propios de la educación. Esta disposición positiva del entorno primario y sus efectos sobre el logro educativo es conceptualizada con el término «Aculturación selectiva» (Aparicio y Portes, 2014), y sus efectos sobre el logro educativo de las segundas generaciones va a depender principalmente de la calidad de las relaciones filio-parentales, sin que el capital humano o el nivel educativo de los progenitores tengan necesariamente un papel decisivo. De este modo es posible explicar el éxito educativo alcanzado por un gran número de hijos de inmigrantes cuyos progenitores poseen un capital humano bajo, sin estudios o con estudios básicos.

En lo que respecta al logro educativo como variable clave para la integración social de los individuos hay, como venimos diciendo, un amplio consenso en la literatura. Si bien, la información sobre el acceso a la universidad de las segundas generaciones en España es muy escasa (una revisión de la literatura hasta 2014 ver en Egido, Fernández y Galán, 2014), cabe destacar el trabajo de Aparicio y Portes (2014). En una muestra de 602 individuos nacidos en España, hijos de padres extranjeros, con 18 años de edad y residentes en Madrid y Barcelona, estos autores encuentran que sólo un 8,1\% cursan estudios universitarios. 
En el mismo estudio, del conjunto de jóvenes con antecedentes migratorios ( $n=3.232$ ), nacidos o no en España, con 18 años de edad y residentes en las mismas ciudades, la proporción de sujetos que accedieron a los estudios universitarios se reducía a un pobre 4,8\% (P. 94).

Sujeto a las diferencias que puedan detraerse de los diferentes modelos de acceso a la universidad, en la literatura hay consenso respecto a la fuerte relación existente entre el acceso a la educación superior y la posición socioeconómica de los estudiantes, y a menudo de la mano, el nivel educativo de los progenitores (Herrera, 2019). En términos generales, los resultados son coincidentes con la tendencia que Bourdieu y Passeron $(1964 ; 2009)$ encontraron en el sector universitario francés en la década de los 60 del siglo xx. Existe una sobrerrepresentación persistente en las aulas universitarias de los estudiantes de clase media.

En Alemania, Riphahn (2003), compara la participación en la educación superior de diferentes cohortes de población con antecedentes migratorios observando una infrarrepresentación persistente de las segundas generaciones en la composición social universitaria. En el ámbito anglosajón, junto a las barreras de género y económicas, autores como Taylor (1992; 1993) suman las relacionadas con el origen étnico. Reay (2001) remite a los estudios de Modood y Acland (1998, en Reay et al., 2001), quienes observan un incremento de matriculaciones universitarias entre las minorías durante la segunda década de los 90 del siglo xx en el Reino Unido a pesar de su perfil ocupacional parental menos favorecido. Entre 1997 y 1998 , la mayoría de grupos étnicos mostraron aumentos en los solicitantes aceptados para cursos de grado, pero hubo un número que había disminuido. La categoría blanca cayó un 3,37 por ciento, pero los solicitantes negros del Caribe y de África negra cayeron casi el doble; 6.01 y 6.75 por ciento respectivamente. Sin embargo, los patrones agregados de entrada de grupos étnicos y de clase solo cuentan una parte de la historia de las desigualdades y el desarrollo de estratificación social dentro de la educación superior. La expansión de mercados empresariales transnacionales de títulos de educación superior en el mundo ha potenciado la expansión de instituciones privadas expendedoras de este tipo de credenciales. Mientras que más estudiantes de clase trabajadora y de minorías ingresan a la universidad, en su mayor parte ingresan a universidades diferentes a sus homólogos de clase media. El modelo mercantil de la educación superior en el Reino Unido es un ejemplo de las tendencias comprendidas en las agendas políticas de los gobiernos neoliberales en el mundo. La división binaria en la educación superior británica ha fomentado el surgimiento de una nueva jerarquía de instituciones en la que prestigiosas universidades de investigación se han convertido en una capa superior de instituciones de élite y son estas universidades las que siguen siendo abrumadoramente de composición blanca y de clase media (Brown, 2013; Bathmaker et al., 2016).

\section{Metodología}

Desgraciadamente, el Censo de población y viviendas realizado en España en 2011 no permite abordar directamente los procesos de aculturación, ya que no ofrece información sobre los procesos de adaptación a la cultura de acogida. Para realizar el estudio comparativo de los predictores estructurales sobre el acceso a la universidad de las segundas generaciones en España, utilizamos los datos sociodemográficos que se facilitan sobre la edad, el sexo, la nacionalidad, el país de nacimiento y el tiempo de residencia en España ${ }^{2}$ de los individuos, además de otros datos de tipo estructural como la situación laboral y el nivel de estudios de los progenitores.

2 Es necesario tener en cuenta que los individuos que, en el año 2011, entonces tenían entre 18 y 21 años de edad conforman la primera cohorte nacida de la corriente migratoria que llegó a España durante los últimos años de la década de los 80 y primeros de la década de los 90 del siglo XX, periodo en el que entraron las primeras olas importantes de trabajadores internacionales a España. 
La técnica estadística utilizada es la Regresión Logística Binaria, técnica analítica que nos permite relacionar funcionalmente una variable dicotómica con un conjunto de variables independientes. El software utilizado para el tratamiento de datos es el Statistical Package for the Social Sciences (SPSS).

En el estudio calculamos la probabilidad de que las segundas generaciones de inmigrantes en España estudien cursen estudios de educación superior en función del grupo étnico de referencia y la situación socioeconómica familiar, respecto de la población autóctona del mismo grupo de edad, entre los 18 y los 21 años, para ello desagregamos las segundas generaciones de inmigrantes atendiendo a las clasificaciones utilizadas en la literatura (Feixa, 2008). En primer lugar, los hijos de los migrantes nacidos en el lugar de destino (la segunda generación propiamente dicha), a quienes dividimos a su vez en dos grupos: por un lado, los nacidos de matrimonios mixtos y, por otro, aquellos nacidos de matrimonios coétnicos. En segundo lugar, los nacidos en la sociedad de origen, pero socializados en la sociedad de acogida, la llamada «generación 1.5». Este grupo se conforma en este estudio por aquellos individuos nacidos en el país de origen pero que llegaron a España a una edad muy temprana, sumando, al menos, diez años de residencia en el país, es decir, que llegaron con entre ocho y once años de edad. Finalmente, la población autóctona, categoría que agrupa a aquellos nacidos en España e hijos de padres a su vez nacidos en España.

Para alcanzar los objetivos de este estudio seleccionamos dos grupos de minorías étnicas con una gran presencia en España: individuos procedentes del Norte de África (Argelia, Túnez, Marruecos y Sahara Occidental) y de América del Sur (Honduras, República Dominicana, Bolivia, Brasil, Colombia, Ecuador, Perú, Venezuela, Uruguay y Argentina). Ambos grupos presentan importantes diferencias y similitudes socioculturales (fundamentalmente, idioma y tradición religiosa) en relación con las características de la sociedad de destino, lo que resulta de gran interés para el análisis comparado con la población autóctona y con la generalidad del conjunto de las segundas generaciones y la generación $1.5^{3}$.

La variable dependiente dicotómica elegida para las regresiones «Cursando estudios universitarios», no incluye los estudios de master y doctorado. Para construir la variable independiente sobre el estatus socioeconómico familiar se han cruzado datos de la situación laboral y los estudios completados de los progenitores. De este modo, construimos seis categorías de contraste: «Trabajan y Estudios de 19» (Ambos progenitores tienen estudios primarios — hasta la ESO_, o no tienen, y ambos, o al menos uno, se encuentran ocupados a tiempo completo); «Desempleo y Estudios de 19 (Ambos tienen estudios primarios, y ambos se encuentran en situación de desempleo); «Trabajany Estudios de 29 (Ambos, o al menos uno, de los progenitores tiene estudios secundarios y ambos, o al menos uno, se encuentra ocupado a tiempo completo); «Desempleo y Estudios de 29》 (Ambos tienen estudios de secundarios y ambos se encuentran en situación de desempleo); «Trabajan y Estudios de 39» (Ambos, o al menos uno, de los progenitores tiene estudios terciarios y ambos, o al menos uno, se encuentra ocupado a tiempo completo); «Desempleo y Estudios de 39» (Ambos tienen estudios terciarios y ambos se encuentran en situación de desempleo).

Respecto a la categoría «edad» de los progenitores introducimos en el análisis la edad de la madre. Esta variable recoge grupos de edad quinquenal a partir de los 25 , edad media al primer hijo entre las mujeres nacidas en el extranjero. Finalmente, la variable «Tamaño familian» recoge diferentes categorías según el número de miembros de convivencia hasta una cantidad igual o mayor de seis.

3 Dado la significatividad de estas características decidimos analizar por separado la generalidad de segundas generaciones y generación 1.5, por un lado, y, por otro, las correspondientes a estas minorías (procedentes de África y de América del sur). 
Las variables independientes categóricas introducidas son las siguientes: «sexo», «edad de la madre», «estado civil de la madre», así como los «estudios completados» $\mathrm{y}$ «situación laboral» del padre y de la madre. Además, introducimos una variable socioeconómica calculada cruzando la situación laboral con los estudios completados del padre y de la madre, dando lugar a seis categorías descritas en el apartado sobre metodología. Finalmente, cada una de las variables independientes dicotómicas introducidas se corresponden con los antecedentes migratorios de los grupos que se analizan, a saber, Autóctonos; $2^{a}$ generación de matrimonios mixtos; $2^{a}$ generación de matrimonios coétnicos; y Generación 1.5. En un segundo paso se introducen en el análisis las $2^{\mathrm{a}}$ generaciones y generaciones 1.5 , Norteafricanas (AN) y Latinoamericanas (AS). La introducción de estas dos últimas categorías nos permite comparar resultados específicos de ambas minorías con los resultados más generales obtenidos en el primer paso.

La categoría de contraste en el caso de la variable «sexo» es «hombre», en el caso de la «edad de la madre» es «mayor 50 años», para el «estado civil de la madre» es «soltera», y, finalmente, para la «situación socioeconómica» la categoría de referencia es «Trabajany Estudios de 1\%» (Ambos progenitores tiene estudios primarios — hasta la ESO_, o no tienen, y ambos, o al menos uno, se encuentra ocupado a tiempo completo).

\section{Resultados}

Como podemos ver en el gráfico 2, la población joven con nacionalidad distinta a la española, con edades comprendidas entre los 18 y los 21 años, representa en torno al $11 \%$ de la población total de este grupo de edad en España. La mayoría procede de la Unión Europea (28,39\%, en 2019), de África (25,6\%, de Marruecos en su mayoría) y de Latinoamérica (29,8\%), todos ellos con tendencia ascendente a lo largo del periodo. También los jóvenes con nacionalidad de origen en Asia presentan una tendencia similar, aunque en proporciones muy inferiores (10,31\%, con origen en China fundamentalmente) (Ver gráfico 2).

\section{Gráfico 2. Distribución de extranjeros residentes en España con edades comprendidas entre los 18 y los 21 años según nacionalidades principales}

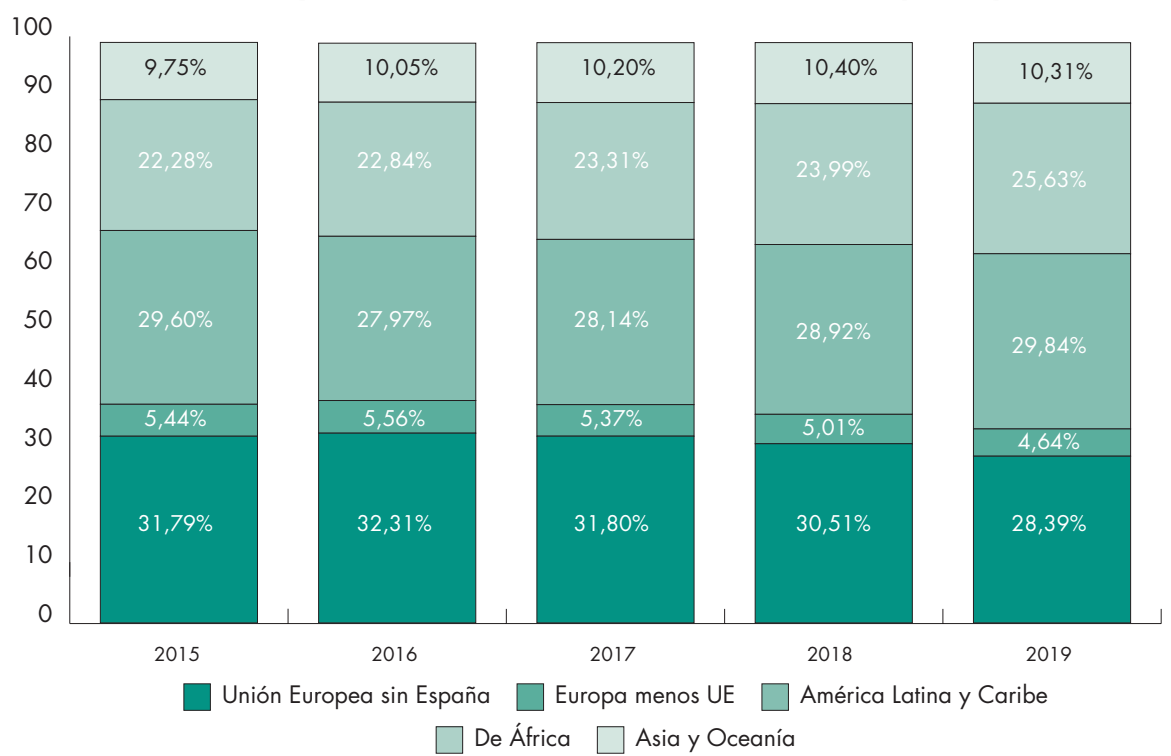

Gráfico de elaboración propia. Fuente: Ministerio de Educación y Formación Profesional (MEFP). 
Incluyendo a los jóvenes españoles, la composición social total del estudiantado de nuevo ingreso en la universidad española presenta notables diferencias si atendemos a su distribución según nacionalidades. Como vemos en el gráfico 3, los jóvenes autóctonos que ingresan a la universidad representan el 15,48\% del total de los jóvenes de igual nacionalidad (española) y mismo grupo de edad.

Con tasas similares encontramos a la población joven con origen en la Unión Europea (14,26\%), resto de Europa $(13,13 \%)$ y en Asia y Oceanía (11,89\%, China principalmente). Con tasas más bajas encontramos a los jóvenes de origen latinoamericano (8,23\%) y africano (4,54\%, Marruecos principalmente). Estudios recientes ya señalan una caída importante de las segundas y primeras generaciones en los estudios de bachillerato (Bayona-i-Carrasco, J., Domingo, A., 2021).

Teniendo en cuenta esta distribución podemos diferenciar dos bloques. Un primer bloque formado por aquellas nacionalidades con tasas de población de nuevo ingreso superiores al 10\% (autóctonos, europeos y asiáticos), y un segundo bloque con tasas inferiores al 10\% (Latinoamérica y África) (Ver gráfico 3). Estas diferencias se hacen mayores si consideramos que los orígenes latinoamericano y africano tienen una mayor representación en la población general como podemos apreciar en el gráfico 2.

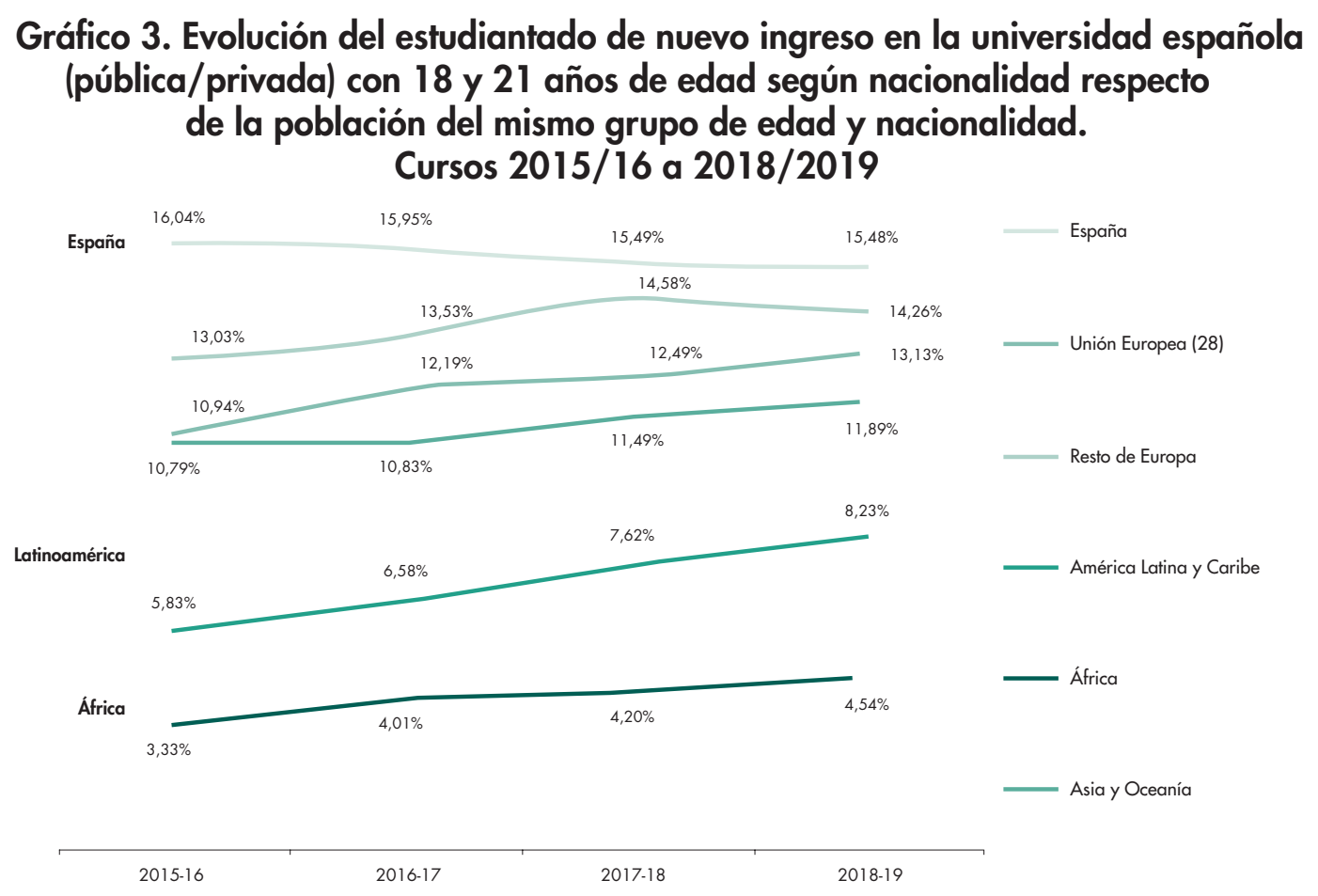

Gráfico de elaboración propia. Fuente: MEFP.

Como indicamos en el apartado introductorio, para estudiar la relación de los antecedentes tanto socioeconómicos como migratorios y el acceso a la universidad, se realizaron una serie de regresiones binarias tomando como variable dependiente dicotómica (sí/no) la categoría «cursando estudios terciarios», indicando con los coeficientes positivos una mayor probabilidad de acceso. Se comienza introduciendo variables objetivas como el sexo, la edad de la madre, los estudios y ocupación de los progenitores, el país de nacimiento, los antecedentes migratorios y el tamaño del hogar. Posteriormente, procedemos en un segundo paso a introducir categorías calculadas a partir del estatus socioeconómico familiar, los antecedentes migratorios y el origen étnico de estos antecedentes. 
El paso nulo proporciona la estimación del término constante $(B=-0,688)$, y una significación fuerte $(0,000)$. Con el 70,8\% de casos clasificados (Ver Anexo I) y un valor de corte de 0,5 , el resumen presenta una buena bondad de ajuste del modelo con un Pseudo R2 de Nagelkerke del 17,7\% (Ver tabla I).

\section{Tabla I. Estimación y estadísticos asociados}

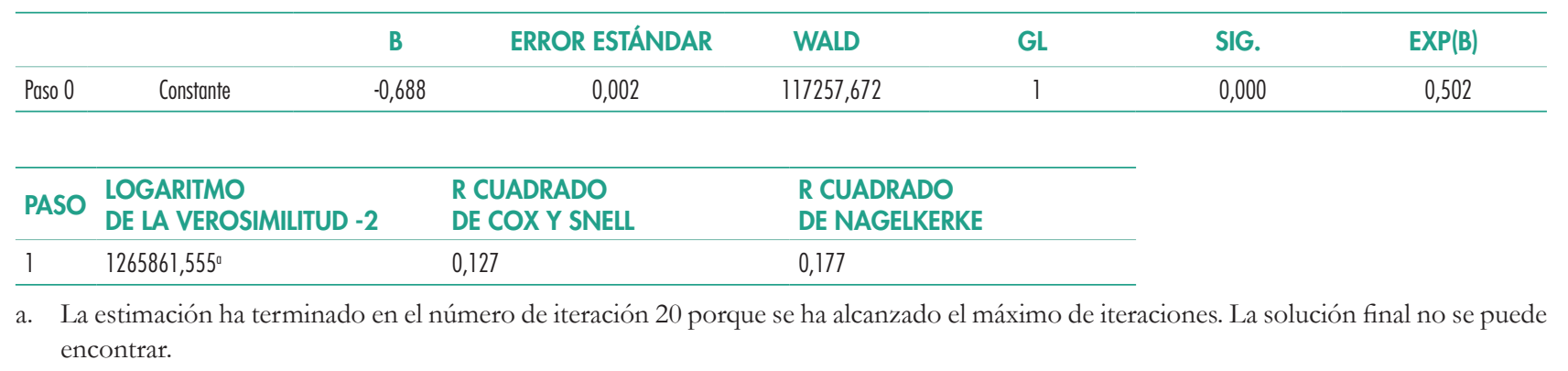

La probabilidad de estar cursando algún estudio de grado universitario muestra una tendencia favorable para las mujeres frente a los hombres. Ellos tienen solo dos tercios de la probabilidad que tienen ellas de ir la universidad. Igualmente, la edad de la madre es significativa. Un aumento en la edad de la madre (> 45 años) incrementa la probabilidad de estar en la universidad.

Respecto al estado civil de los padres, la «pareja intacta» incrementa la probabilidad de que los hijos accedan a la universidad respecto a estar «soltera» (31,3\% en el caso de las mujeres casadas y el 26,8\% en el caso de los hombres casados), sin embargo, se producen diferencias significativas en el resto de las figuras (separada, divorciada o viuda) en función de si éstas se refieren al padre o a la madre. En el caso de la madre, los estados «separada», «divorciada» o «viuda» reducen la probabilidad de que los hijos se encuentren cursando estudios superiores. En el caso del padre, por el contrario, no sólo la aumentan, con excepción de estado de viudez, sino que lo hacen en mayor medida incluso que en el caso de encontrarse «casado». En relación con el tamaño familiar, los resultados señalan un aumento de la probabilidad de cursar estudios entre los individuos pertenecientes a núcleos familiares compuestos de más de dos miembros (Ver tabla II).

Los estudios terciarios, tanto del padre como de la madre, son buenos predictores. En este sentido, la probabilidad de estar cursando estudios universitarios disminuye en la medida que baja el nivel educativo de los progenitores. Igualmente, respecto al efecto de la situación laboral, el que alguno de los progenitores, o ambos, se encuentre «ocupado a tiempo completo» aumenta la probabilidad de ir a la universidad.

Respecto a la predictores migratorios, el estar cursando estudios universitarios y no tener antecedentes migratorios tiene una relación estadística fuerte y positiva. En menor grado, pero en la misma dirección, la probabilidad de cursar este tipo de estudios por parte de los jóvenes nacidos en España e hijos de matrimonios mixtos, también es fuerte y positiva. Por el contrario, haber nacido en España, o fuera de España, en el seno de matrimonios coétnicos reduce considerablemente la probabilidad de encontrarse estudiando en la universidad (Ver tabla II). 
Tabla II. Resultados Regresión L. Binaria (a)

\begin{tabular}{|c|c|c|c|}
\hline & B & SIG. & $\operatorname{EXP}(B)$ \\
\hline Sexo 1/6 (Hombre) & $-0,385$ & 0,000 & 0,680 \\
\hline Edad de la Madre (35-39) & 0,480 & 0,001 & 1,616 \\
\hline Edad de la Madre (40-44) & 0,826 & 0,000 & 2,283 \\
\hline Edad de la Madre (45-49) & 1,121 & 0,000 & 3,068 \\
\hline Edad de la Madre $(>50)$ & 1,047 & 0,000 & 2,850 \\
\hline E. Civil de la Madre(Casada) & 0,283 & 0,000 & 1,327 \\
\hline E.C. de la Madre (Separada) & $-0,081$ & 0,243 & 0,923 \\
\hline E.C. de la Madre (Divorciada) & $-0,034$ & 0,410 & 0,967 \\
\hline E. Civil del Padre (Casado) & 0,237 & 0,000 & 1,268 \\
\hline E.C. del Padre (Separado) & 0,527 & 0,000 & 1,694 \\
\hline E.C. del Padre (Divorciado) & 0,302 & 0,000 & 1,352 \\
\hline T. Familiar (3 personas) & 1,436 & 0,000 & 4,206 \\
\hline T. Familiar (4 personas) & 1,518 & 0,000 & 4,565 \\
\hline T. Familiar (5 personas) & 1,463 & 0,000 & 4,320 \\
\hline T. Familiar ( 26 personas) & 1,401 & 0,000 & 4,061 \\
\hline Estudios del Padre ( $\leq$ ESO) & $-0,994$ & 0,000 & 0,370 \\
\hline E. del Padre (Secundaria) & $-0,603$ & 0,000 & 0,547 \\
\hline Estudios de la Madre ( $\leq$ ESO) & $-1,141$ & 0,000 & 0,320 \\
\hline E. de la Madre (Secundaria) & $-0,571$ & 0,000 & 0,565 \\
\hline SL Padre (Ocup. T. Completo) & 0,329 & 0,000 & 1,389 \\
\hline SL Madre (Ocup. T. Completo) & 0,154 & 0,000 & 1,166 \\
\hline (S) A.M. (Autóctonos) & 0,596 & 0,000 & 1,815 \\
\hline A.M. $2^{\circ}$ G. Matrimonio Mixto & 0,464 & 0,000 & 1,590 \\
\hline A.M. $2^{\circ}$ G. Matrimonio Coétnico & $-0,026$ & 0,392 & 0,975 \\
\hline A.M. Generación 1.5 & $-0,424$ & 0,000 & 0,655 \\
\hline
\end{tabular}

Como señalamos en el apartado dedicado al procedimiento metodológico seguido en este trabajo, junto con las variables anteriores hemos introducido una variable calculada a partir de la situación laboral de ambos progenitores y el nivel de estudios alcanzados por éstos, de este modo, podemos conocer el efecto que sobre la probabilidad de cursar estudios universitarios en función de los antecedentes migratorios tiene el origen socioeconómico de los individuos.

Los resultados nos muestran que, en el caso de la población autóctona (sin antecedentes migratorios), la probabilidad de cursar estudios universitarios es fuerte y positiva cuando media un capital educativo familiar alto (Estudios terciarios) (Ver tabla III). Esta tendencia se invierte cuando el capital educativo disminuye (Secundaria y Primarios). En los casos en que los progenitores poseen estudios de la ESO o de secundaria postobligatoria, el efecto de los estudios se diluye en favor de la situación laboral, variable que pasa a convertirse en el factor más influyente. Los hijos cuyos padres tienen estudios de secundaria postobligatoria y se encuentran trabajando (al menos uno de ellos) presentan menos probabilidad de estar cursando estudios universitarios que los hijos cuyos padres tienen estudios hasta la ESO e igualmente se encuentran trabajando (al menos uno de ellos) (Ver tabla III).

En principio, este resultado parece contra-intuitivo. Obviamente, en este caso, el nivel de estudios no parece ser el factor que determina la orientación hacia la universidad de los estudiantes. Para explicar esto es preciso aclarar que la variable calculada no nos permite saber si la condición «Empleado a tiempo com- 
pleto» se cumple para un progenitor o para ambos, por lo que es plausible pensar que la variación entre una situación u otra determine los valores de ambas categorías en los resultados de la ecuación. Es decir, si la condición «Ocupado a tiempo completo» se da en los dos progenitores, ambos con estudios primarios (categoría de referencia), se hace factible pensar que la probabilidad de que este grupo pueda enviar a sus hijos a la universidad sea mayor que la situación en la que dicha condición se dé en un solo progenitor, independientemente del nivel de estudios. Esto nos permite deducir que la situación económica familiar tiene un efecto importante sobre la decisión de los padres de enviar a sus hijos a la universidad.

En necesario hacer mención también en este apartado a otra situación contra-intuitiva que se produce, especialmente, entre las segundas generaciones. Nos referimos al hecho de que la probabilidad de cursar estudios superiores entre los estudiantes en desventaja socioeconómica y capital educativo familiar bajo (secundaria no obligatoria o primaria) es significativa entre los estudiantes con antecedentes migratorios, especialmente entre las segundas generaciones, independientemente del grupo étnico de pertenencia. Tal resultado nos lleva a ponerlo en correspondencia con el efecto que autores como Aparicio y Portes (2014) encuadran bajo el concepto de Aculturación selectiva, el cual, responde a la existencia en el entorno primario de una disposición positiva hacia los valores de la educación que motiva al joven inmigrante hacia el logro educativo. La riqueza de esta conceptualización, por tanto, permitiría también explicar aquellos casos en los que las segundas generaciones alcanzan buenos resultados educativos sin que el capital humano o el nivel educativo de los progenitores tengan un papel explicativo decisivo.

En este sentido, una vez introducidas las variables étnicas y socioeconómicas encontramos que las segundas generaciones de matrimonios coétnicos (norteafricanos y suramericanos) presentan, en términos generales, probabilidades significativas de cursar estudios terciarios. Como podemos ver en la tabla III, estas probabilidades son similares en las segundas generaciones de norteafricanos y suramericanos, tanto entre cuyos padres, con estudios hasta la ESO, no se encuentran ocupados a tiempo completo, como entre aquellos cuyos padres, con estudios secundarios o terciarios, se encuentran trabajando. Cabe destacar, entre los primeros, la sobresaliente fortaleza que muestra la probabilidad de cursar estudios universitarios entre las segundas generaciones de suramericanos con un bajo capital educativo familiar.

En el caso de la generación 1.5 (nacidos fuera de España con al menos diez años de residencia en el país), algunas de las categorías no entran en la ecuación debido al bajo número de casos que presentan. Es el caso de los norteafricanos cuando al menos uno de los progenitores tiene estudios terciarios y trabaja o cuando poseen estudios hasta la ESO y no trabajan; y el de los suramericanos cuando al menos uno de los progenitores no trabaja y posee, o bien estudios primarios o bien estudios terciarios (sí entran los que poseen estudios secundarios) (Ver tabla III).

Los resultados obtenidos en las categorías que sí entran en el modelo muestran resultados similares a los obtenidos para las segundas generaciones (jóvenes nacidos en España de matrimonios coétnicos). La probabilidad de cursar estudios superiores aumenta cuando la posición socioeconómica familiar es buena (padres con estudios terciarios, o de secundaria en el caso de los norteafricanos, en situación de «empleados a tiempo completo») y se debilita (en el caso de los norteafricanos) o se reduce (en el caso de los suramericanos) cuando la posición socioeconómica familiar disminuye. 
Tabla III. Resultados Regresión L. Binaria (b)

\begin{tabular}{|c|c|c|c|}
\hline & B & SIG. & $\operatorname{EXP}(B)$ \\
\hline \multicolumn{4}{|l|}{ Autóctonos } \\
\hline Parados/Est. 2 $2^{0}$ (Secundaria Post.) Autóctonos & $-0,183$ & 0,000 & 0,833 \\
\hline Ocupados/Est. $2^{0}$ Autóctonos & $-0,072$ & 0,001 & 0,930 \\
\hline Ocupados/Est. 3os Autóctonos & 0,188 & 0,000 & 1,206 \\
\hline Parados/Est. $1^{\text {os }}$ Autóctonos & $-0,032$ & 0,266 & 0,969 \\
\hline Parados/Est. 3os Autóctonos & 0,171 & 0,000 & 1,186 \\
\hline \multicolumn{4}{|c|}{$2^{a}$ G. M. coétnico Norte de África (NA) } \\
\hline Parados/Est. $2^{a} 2^{a} \mathrm{G}$. (NA) & $-0,089$ & 0,443 & 0,915 \\
\hline Ocupados/Est. $2^{0} 2^{0} \mathrm{G}$. (NA) & 0,130 & 0,004 & 1,138 \\
\hline Ocupados/Est. 3os $2^{\circ} \mathrm{G}$. (NA) & 0,136 & 0,004 & 1,146 \\
\hline Parados/Est. $\operatorname{los} 2^{\circ} \mathrm{G}$. (NA) & 0,399 & 0,022 & 1,490 \\
\hline Parados/Est. 3os $2^{0} \mathrm{G}$.(NA) & 0,695 & 0,142 & 2,005 \\
\hline \multicolumn{4}{|c|}{$2^{a}$ G. M. coétnico Sur América (SA) } \\
\hline Parados/Est. $2^{a} 2^{a} G$. (SA) & 0,196 & 0,031 & 1,216 \\
\hline Ocupados/Est. $2^{0} 2^{0} \mathrm{G}$. (SA) & 0,366 & 0,000 & 1,441 \\
\hline Ocupados/Est. $3^{\circ \text { os }} 2^{0} G$. (SA) & 0,380 & 0,000 & 1,462 \\
\hline Parados/Est. 1os $2^{\circ} G$. (SA) & 2,449 & 0,000 & 11,575 \\
\hline Parados/Est. $3^{\circ s} 2^{\circ} \mathrm{G} .(S A)$ & 0,317 & 0,083 & 1,373 \\
\hline \multicolumn{4}{|c|}{ 1.5 G. M. coétnico Norte de África (NA) } \\
\hline Parados/Est. $2^{\circ} \mathrm{G} .1 .5$ (NA) & 1,066 & 0,003 & 2,903 \\
\hline Ocupados/Est. $2^{\circ} \mathrm{G} .1 .5$ (NA) & 2,050 & 0,000 & 7,766 \\
\hline Parados/Est. 3os G. 1.5 (NA) & 0,990 & 0,000 & 2,692 \\
\hline \multicolumn{4}{|l|}{ 1.5 G. M. coétnico Sur América (SA) } \\
\hline Parados/Est. $2^{\circ}$ G. 1.5 (SA) & $-1,301$ & 0,000 & 0,272 \\
\hline Ocupados/Est. $2^{\circ}$ G. 1.5 (SA) & 0,642 & 0,000 & 1,899 \\
\hline Ocupados/Est. 3os G. 1.5 (SA) & 0,319 & 0,000 & 1,376 \\
\hline
\end{tabular}

\section{Conclusiones}

En este trabajo hemos estudiado el efecto de factores socioeconómicos familiares, como el nivel de estudios y la situación laboral de los progenitores, sobre la probabilidad de cursar estudios universitarios de grado (no se incluyen estudios de master y doctorado) de las cohortes nacidas entre 1987 y 1993 residentes en España y que en 2011 contaban con edades comprendidas entre los 18 y los 21 años, atendiendo a la existencia o no de antecedentes migratorios y la pertenencia a uno de los siguientes grupos étnico-culturales: autóctonos, norteafricanos y suramericanos.

Respondiendo a este planteamiento inicial hemos comprobado que hay una relación fuerte y positiva entre los factores socioeconómicos y el cursar estudios terciarios. Una buena posición socioeconómica aumenta la probabilidad de acceder a la universidad para todos los grupos. En sentido contrario, hemos observado que esta probabilidad se reduce considerablemente entre las segundas generaciones nacidas de matrimonios coétnicos, hayan nacido dentro ( $2^{\mathrm{a}}$ generación) o fuera (generación 1.5) de España, lo que evidencia la existencia de segregación en el acceso a la universidad de estos grupos.

Por la metodología empleada en esta investigación no podemos extraer conclusiones acerca de los factores socioculturales que pudieran estar ejerciendo algún tipo de influencia sobre las oportunidades y motivaciones de los jóvenes con antecedentes migratorios a la hora de acceder a la universidad, pero 
pensamos que tiene interés detenernos a examinar alguno de los resultados obtenidos que, en trabajos precedentes, han sido asociados a este tipo de factores (Borjas,1992; Portes y De Wind, 2004; Aparicio y Portes, 2014). Nos referimos a la cualidad de las actitudes positivas hacia la integración social a través de la educación asociadas a determinadas comunidades minoritarias (Borjas, 1992) o a los entornos primarios étnicos de pertenencia de los individuos (Aparicio y Portes, 2014).

Al igual que Modood y Acland (1998, en Reay et al., 2001), en el ámbito del Reino Unido, observaron un aumento de las matriculaciones universitarias de las segundas generaciones en la década de los noventa. Los resultados obtenidos en nuestro análisis sugieren la posibilidad de encontrar una evolución similar en España durante la primera década del siglo xxi. Las cohortes de segundas generaciones de matrimonios coétnicos (norteafricanos y suramericanos) con una posición socioeconómica inferior (progenitores con estudios primarios, o secundarios, en situación de desempleo), nacidas entre 1987 y 1993, y, por tanto, con edades comprendidas entre los 18 y los 21 años en 2011, presentaban frecuencias de acceso a la universidad bajas.

Igualmente, Aparicio y Portes (2014) encontraron casos similares en Madrid y Barcelona. Desde el punto de vista de estos autores, los casos señalados responden a la existencia de una disposición positiva hacia los valores de la educación por parte del entorno primario que motiva al joven inmigrante hacia el logro educativo (acceso a la educación superior en nuestro caso). Esta motivación explícita esta conceptualizada bajo el término de «Aculturación selectiva» (Aparicio y Portes, 2014). Su influencia en los resultados educativos de las segundas generaciones va a depender principalmente de la calidad de las relaciones entre padres e hijos, sin que la ocupación o la educación de los progenitores tengan necesariamente un papel determinante.

Desde nuestro punto de vista, esta disposición positiva hacia los valores de la educación es un buen instrumento para explicar el éxito educativo alcanzado por un número importante de hijos de inmigrantes cuyos progenitores poseen un capital humano bajo, sin estudios, o con estudios básicos, y malos empleos. Ciertamente, estas actitudes motivacionales positivas hacia la educación superior pueden responder a un interés intrínseco hacia el saber y el conocimiento que los individuos se preocupan de transferir a las siguientes generaciones, bien a un pragmatismo orientado por el interés extrínseco hacia la mejora y la prosperidad económica intergeneracional, o bien a una combinación de ambos intereses, sin embargo, en cualquiera de estos casos, a nuestro parecer, se nos hace difícil asociar esta disposición positiva a una u otra minoría.

\section{Referencias bibliográficas}

Alba, Richard (2005): "Bright vs Blurred Boundaries: Second-Generation Assimilation and Exclusion in France, Germany, and the United States". Ethnic and Racial Studies, 28 (1), 20-49. DOI: https:/ doi.org/ 10.1080/0141987042000280003.

Álvarez-Sotomayor, Alberto y Martínez-Cousinou, Gloria (2020): “Inmigración, lengua y rendimiento académico en España. Una revisión sistemática de la literatura”. Revista Internacional de Sociología, 78 (3), e160. DOI: https://doi.org/10.3989/ris.2020.78.3.19.083.

Aparicio, Rosa (2007): “The Integration of the Second and 1.5 Generations of Moroccan, Dominican and Peruvian Origin in Madrid and Barcelona". Journal of Ethnic and Migration Studies, 33, 1169-1193. DOI: https:/ / doi.org/10.1080/13691830701541713. 
Aparicio, Rosa y Portes A. (2014). Crecer en España: La integración de los hijos de inmigrantes. Colección Estudios Sociales Núm. 38. Barcelona. Obra Social La Caixa. Disponible en: https://obrasociallacaixa.org/documents/10280/240906/vol38_es.pdf/1a8a03c9-e39a-4853-b15b-bb856989e7d4.

Bathmaker, Ann-Marie; Ingram, Nicola y Waller, Richard (2013): "Higher Education, Social Class and the Mobilisation of Capitals: Recognising and Playing the Game". British Journal of Sociology of Education, 34(5-6), 723-743. DOI: https://doi.org/10.1080/01425692.2013.816041.

Bayona-i-Carrasco, Jordi y Domingo, Andreu (2021): "La continuidad en el aula: el caso del alumnado de origen inmigrante en la transición hacia la educación posobligatoria en Cataluña”. Aposta. Revista de Ciencias Sociales, 89, 123-141, http://apostadigital.com/revistav3/hemeroteca/jbayona.pdf.

Bonal, Xavier (2016): “Crisis, educación y desigualdad: una cuestión de paradojas”. Investigar em Educação, 2(3). Disponible en: http://pages.ie.uminho.pt/inved/index.php/ie/article/view/86.

Borjas, George J. (1992): "Ethnic Capital and Intergenerational Mobility". Quarterly Journal of Economic, 107 (1), 123-150. DOI: https://doi.org/10.2307/2118325.

Bourdieu, Pierre y Passeron, Jean-Claude. (2009). Los herederos: los estudiantes y la cultura. Siglo XXI Editores, 1964.

Brown, Phillip (2013): "Education, opportunity and the prospects for social mobility". British Journal of Sociology of Education, 34(5-6), 678-700. DOI: https://doi.org/10.1080/01425692.2013.816036.

Brubaker, Rogers (1992). Citizenship and Nationhood in France and Germany. Harvard University Press.

Brubaker, Rogers (2001): "The Return of Assimilation? Changing Perspectives on Immigration and its Sequels in France, Germany and the United States". Ethnic and Racial Studies, 24, 531-548. DOI: https:/ / doi.org/10.1080/01419870120049770.

Checa, Juan Carlos y Monserrat, Monserrat (2015): "La integración social de los hijos de inmigrantes africanos, europeos del este y latinoamericanos: Un estudio de caso en España". Universitas Psychologica, 14 (2), 475-486. DOI: https://doi.org/10.11144/Javeriana.upsy14-2.lish.

Checa, Juan Carlos y Arjona, Ángeles (2009): “La integración de los inmigrantes de «segunda generación» en Almería. Un caso de pluralismo fragmentado". Revista Internacional de Sociología, 67 (3), 701-727. DOI: https://doi.org/10.3989/ris.2008.04.17.

Consejo económico y social de España (2020). Informe sobre la inmigración en España: efectos y oportunidades, 2019. Ministerio de Empleo y Seguridad Social. Madrid. Disponible en: http://www.ces.es/documents/10180/5209150/Inf0219.pdf.

Crul, Maurice y Vermeulen, Hans (2006): "Immigration, Education, and the Turkish Second Generation in Five European Nations: A comparative Study" en Craig A. Parsons y Timothy M. Smeeding (eds.): Immigration and the Transformation of Europe. Cambridge: Cambridge University Press. DOI: https://doi. org/10.1017/CBO9780511493577.009. 
Egido Gálvez, Inmaculada; Fernández, Ma José y Galán, Arturo (2014): “La dimensión social del proceso de Bolonia: Apoyos y servicios para grupos de estudiantes poco representados en las universidades españolas”. Educación XX1, 17 (2), 57-81. DOI: https://doi.org/10.5944/educxx1.17.2.11479.

Feixa, Carles (2008): “Generación uno punto cinco". Revista de Estudios de Juventud, 80, 115-127. Disponible en: http://www.pymesonline.com/uploads/tx_icticontent/R02323_generacion.pdf.

Gordon, Milton M. (1964). Assimilation in American Life: The Role of Race, Religion and National Origins. Oxford University Press.

Gualda, Estrella (2007): "Researching Second Generation in a Transitional, European and Agricultural Context of Reception of Immigrants. Working paper series". The Center for Migration and Development: Princeton University. Resumen disponible en: https://ideas.repec.org/p/pri/cmgdev/wp0701.pdf.html.

Herrera Cuesta, Damián (2017): “Empleabilidad versus sobrecualificación. Desajuste entre formación y empleo en las trayectorias laborales de los jóvenes titulados en España”. Sociología del trabajo, (89), 29-52. Disponible en: https://revistas.ucm.es/index.php/STRA/article/view/59635/4564456546827.

Herrera Cuesta, Damián (2019): “¿Quién estudia en la universidad? La dimensión social de la universidad española en la segunda década del siglo XXI”. Revista de Sociología de la Educación-RASE, 12 (1), 7-23. DOI: http://dx.doi.org/10.7203/RASE.12.1.13177.

Instituto Nacional de Estadística (2020). Censo de Población y Viviendas 2011. Madrid. Instituto Nacional de Estadística. Disponible en: https://www.ine.es/dynt3/inebase/es/index.htm?padre=1894\&capsel=1895.

Instituto Nacional de Estadística (2020). Cifras de población, series desde 2002. Instituto Nacional de Estadística (INE). Disponible en: https://www.ine.es/dyngs/INEbase/es/operacion.htm?c=Estadistica_C\&cid $=1254736176992 \&$ menu $=$ resultados\&idp $=1254735572981 \#$ !tabs-1254736195710.

Navas, Marisol; Rojas, Antonio J.; Pumares, Pablo; Lozano, Óscar M. y Cuadrado, Isabel (2010): “Acculturation Profiles by Relative Acculturation Extended Model: Host people, Romanian and Ecuadorian Immigrants". International Journal of Social Psychology, 25 (3), 295-312. DOI: https://doi. org/10.1174/021347410792675624.

Organización Internacional para las Migraciones (2020). Informe sobre las Migraciones en el Mundo 2020. Disponible en: https://publications.iom.int/books/informe-sobre-las-migraciones-en-elmundo-2020.

Portes, Alejandro y DeWind, Josh (2004): “A Cross-Atlantic Dialogue: the Progress of Research and Theory in the Study of International Migration". International Migration Review, 38 (3), 828-851. DOI: https://doi.org/10.1111/j.1747-7379.2004.tb00221.x.

Rahona, Marta y Morales, Susana (2013). Educación e inmigración en España: desafíos y oportunidades. Organización de los Estados Iberoamericanos para la educación, la ciencia y la cultura (OEI).

Reay, Diane; Davies, Jacqueline; David, Miriam y Ball, Stephen J. (2001): “Choices of Degree or Degrees of Choice? in Class, 'Race' and the Higher Education Choice Process”. Sociology, 35 (4), 855-874. DOI: https://doi.org/10.1017/S0038038501008550. 
Riphahn, Regina T. (2003): "Cohort Effects in the Educational Attainment of Second Generation Immigrants in Germany: An Analysis of Census Data". Journal of Population Economics, V.16, 711-737. DOI: https://doi.org/10.1007/s00148-003-0146-1.

Taylor, Paul (1992): "Ethnic Group Data and Applications to Higher Education". Higher Education Quarterb, 46, 359-374. DOI: https://doi.org/10.1111/j.1468-2273.1992.tb01608.x.

Taylor, Paul (1993): "Minority Ethnic Groups and Gender in Access to Higher Education". New Community, 19 (3), 425-440. DOI: https://doi.org/10.1080/1369183X.1993.9976375.

\section{Nota biográfica}

Damián Herrera Cuesta es doctor en Sociología, profesor tutor de la Universidad Nacional de Educación a Distancia. Es miembro del grupo de investigación Estudios sobre Sociedad y Política (UCM-UNED). Sus principales áreas de investigación son la Sociología de la Educación (La dimensión social educativa) y la Sociología política (la participación política). 